\title{
GESTÃO DA MANUTENÇÃO NA INDÚSTRIA 4.0
}

Heitor Cavalari Zanela -heitor.zanela@alunos.unis.edu.br Luciano Costa da Silva Filho - luciano.filho1@alunos.unis.edu.br Wilmar Machado Filho - wilmar.filho@alunos.unis.edu.br Patrícia Werneck Silva de Oliveira - paticso@yahoo.com.br Paulo Henrique Silva Lopes - paulohenrique022@hotmail.com

\section{RESUMO}

Este trabalho visa descrever os procedimentos e ferramentas existentes na indústria convencional, e a transição que vem ocorrendo em virtude das tecnologias emergentes em desenvolvimento no mercado para o cenário da indústria 4.0. A elaboração deste estudo comparativo consiste em conceituar o tema em questão de maneira objetiva, expondo as tecnologias vigentes no setor secundário atual em paralelo com a quarta revolução industrial. Nestas circunstâncias, a gestão da manutenção está em foco, visto que o dinamismo e confiabilidade da linha produtiva depende exclusivamente da preservação de seus maquinários e processos, o gerenciamento e planejamento deste setor deve estar em constante evolução. Tecnologias como Realidade aumentada, nuvem de dados, internet das coisas e big data são tecnologias responsáveis por versatilizar o futuro da indústria.

Palavras chave: Gestão da manutenção, Indústria 4.0, tecnologias emergentes.

\begin{abstract}
This work aims to describe the procedures and tools existing in the conventional industry, and the transition that has been occurring due to the emerging technologies under development in the market to the scenario of industry 4.0. The elaboration of this comparative study consists of conceptualizing the subject in question in an objective way, exposing the technologies in force in the current secondary sector in parallel with the fourth industrial revolution. In these circumstances, maintenance management is in focus, since the dynamism and reliability of
\end{abstract}


the production line depends exclusively on the preservation of its machinery and processes, the management and planning of this sector must be constantly evolving. Technologies such as augmented reality, cloud, internet of things and big data are technologies responsible for making the future of the industry more versatile.

Keywords: Maintenance management, Industry 4.0, emerging technologies.

\section{INTRODUÇÃO}

As tecnologias emergentes são responsáveis pela evolução do mundo tecnológico e econômico, seja pelo fator tecnológico ou econômico. Entretanto sua consolidação prática ainda se encontra em desenvolvimento, sendo um grande desafio para o mercado, cada vez mais complexo e competitivo, que pressiona indústrias e empreendedores a buscarem por inovação imediata. Os avanços vão ser diversificados nos vários segmentos tecnológicos, e os recursos demandados serão amplamente utilizados no cotidiano da sociedade global.

As Revoluções Industriais (RIs) definiram os períodos de transição da vida humana (KON, 2015). A primeira RI (posicionada temporalmente entre 1760 e 1840), marcada pela invenção da máquina a vapor, propiciou a produção mecânica; a segunda iniciou-se no final do século XIX, com o advento da eletricidade e viabilizou a produção em massa por meio, particularmente, das linhas de montagem (KON, 2015). O ciclo digital, ou do computador (terceira RI), apresenta como marco inicial a década de 1960 e foi estimulado a partir do desenvolvimento dos semicondutores, da computação pessoal e da internet (KON, 2015). A Indústria 4.0 surge subsequente à terceira RI - as máquinas ainda necessitavam de profissionais específicos para notificarem e corrigirem seus erros e até para serem ligadas - e é também chamada de quarta RI (MENELAU, 2020). O elemento diferenciador principal entre essas duas últimas revoluções se baseia menos nas ferramentas digitais disponíveis (visto que em ambas é possível visualizá-las, ainda que em estágios evolutivos significativamente distintos) e mais, de maneira central, nas diferentes interações entre as categorias tecnológicas abarcadas pela Indústria 4.0 (MENELAU, 2020).

As indústrias buscam continuamente a modernização e aperfeiçoamento de sua gestão, com o objetivo de aumentar sua produtividade e reduzir os custos envolvidos no processo, tendo a manutenção como influenciadora direta , assim como afirma Lima (2019) “ uma vez 
que garantindo que a manutenção é efetuada de uma forma eficiente, isso significa que um dado equipamento ou sistema terá uma maior disponibilidade produtiva, levando a que a organização atinja melhores resultados". O plano de manutenção adotado pela empresa vai garantir o sucesso da confecção e finalização de seu produto.

Segundo Dias (2017), o debate em torno da indústria 4.0 no Brasil ainda é restrito a algumas áreas e aplicações, existindo, portanto, poucas iniciativas do governo ou associações de classe, o que dificulta a busca por novas atualizações e conhecimentos tecnológicos. De acordo com Silva (2018) "São inúmeras as possibilidades oriundas deste novo paradigma para o setor de manutenção no aumento da qualidade de todo o contexto de suas operações, desde a gestão em suas atividades burocráticas até as atividades técnicas e especialistas.” ou seja, o planejamento e a execução dos serviços de manutenção, impactam diretamente na produção fabril.

Neste artigo, será abordado um estudo comparativo sobre os mecanismos essenciais para o desenvolvimento da gestão da manutenção no setor industrial com relação às tecnologias emergentes originadas pela quarta revolução industrial.

\section{METODOLOGIA}

O estudo consiste em realizar a demonstração do cenário em que o uso da gestão da manutenção estará empregue na indústria de um futuro não tão distante, sendo desenvolvido através de consulta em artigos científicos atuais que reforçam e engrandecem a importância de uma empresa investir em pesquisas tecnológicas voltadas para área da informação, pilar fundamental que $4^{\circ}$ revolução industrial oferece para a manutenção das tecnologias emergentes.

Um comparativo será traçado entre os procedimentos existentes para o gerenciamento da manutenção em "chão de fábrica", que se encontra relativamente defasado com o atual cenário global, em comparação ao que já está sendo desenvolvido e testado com relação a implementação de tecnologias versáteis que facilitam e auxiliam no cotidiano do gestor para com o dinamismo de sua empresa.

Portanto,a pesquisa acadêmica e a exemplificação teórica do comparativo entre as ferramentas gerenciais, vão conduzir o desenvolvimento do artigo relatando com detalhes os efeitos desta tecnologia emergente. 


\section{ESTUDO COMPARATIVO}

\section{Planejamento e controle da manutenção - PCM e a Gestão da manutenção}

Em um mundo cada vez mais globalizado e competitivo, em que os consumidores estão sempre buscando por produtos de alta qualidade e de baixo custo, as indústrias tiveram que no decorrer dos anos adequar seus processos produtivos em prol da demanda mundial, sendo moldadas de acordo com o período histórico vigente. Neste cenário, criou-se o Planejamento de Controle de Manutenção (PCM), com a finalidade de proporcionar aos maquinários e sistemas industriais ferramentas e procedimentos de gestão visando a análise e solução de problemas em ambiente fabril (CAMPOS, 2020). A figura 1 demonstra o posicionamento do PCM na estrutura organizacional da empresa.

Figura 1: O PCM na empresa.

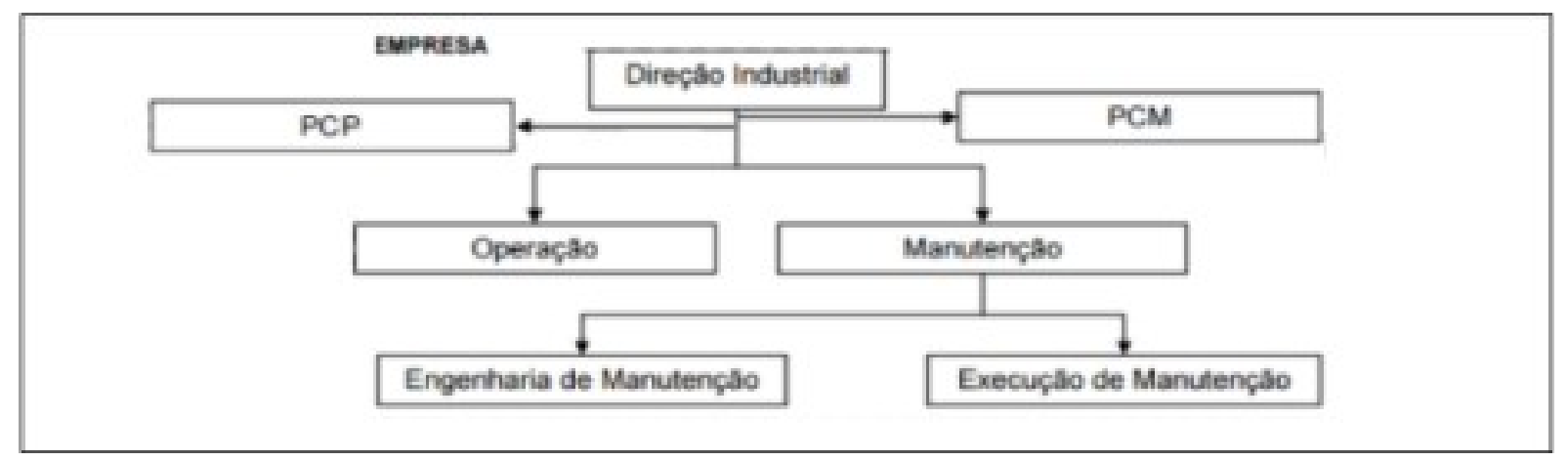

Fonte: Adaptada pelos autores.

Filho (2008) caracteriza o PCM como "conjunto de ações para preparar, programar, verificar o resultado da execução das tarefas de manutenção contra valores preestabelecidos e adotar medidas de correção de desvios para a consecução dos objetivos e da missão da empresa, usando os meios disponíveis.". Campos (2020) propõe que o PCM tem por responsabilidade definir a periodicidade dos planos de manutenção bem como os procedimentos existentes nas OS's ( Ordens de serviços) a serem verificados pelos técnicos de campo durante a realização do serviço. É importante que o setor opere em alinhamento com a gerência e a disponibilidade do setor de PCP ( Planejamento de controle de produção) na criação e revisão da programação das manutenções, visando sempre a menor perda de produtividade por conta de tempo de máquina parada.

Campos (2020) acrescentam que "o PCM fortalece o ciclo de gerenciamento da 
manutenção em uma organização". Em que as informações cotidianas presentes nas O.S. com relação a máquina, tempo de execução, componente defeituoso, sintoma apresentado, causa do defeito, serviço realizado e técnico responsável, serão levantadas e trabalhadas pelo setor com o intuito de aperfeiçoar a gestão na empresa. "Gestão de manutenção tem como estratégia estabelecer novos padrões, mudar os já existentes e cumpri-los, dentro deste contexto as atividades padronizadas de manutenção tende a buscar sempre a excelência das empresas" (CAMPOS,2020). A figura 2 demonstra um modelo de ordem de serviço elaborada pelos autores com base nas revisão de literatura.

Figura 2: Ordem de serviço.

\begin{tabular}{|c|c|c|c|}
\hline \multicolumn{4}{|c|}{ Ordem de serviço } \\
\hline MiQCTNA: & $\operatorname{xxxx}$ & SETOR EXECUTANTE & $\mathbf{x x x}$ \\
\hline \multicolumn{4}{|c|}{ DESCRIÇĊO DO SERITÇO } \\
\hline \multicolumn{4}{|c|}{ 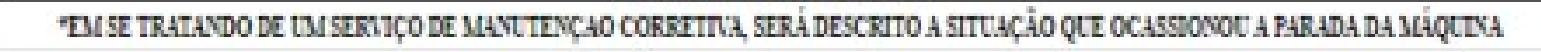 } \\
\hline \multicolumn{4}{|c|}{ 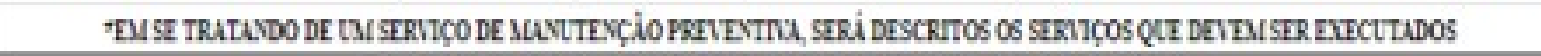 } \\
\hline \multicolumn{4}{|c|}{ ASSINATCRA DOS RESPONSAIEIS PELA EXECTCC.OO DO SERTTCCO } \\
\hline \multicolumn{2}{|c|}{$\operatorname{xxxx}$} & \multicolumn{2}{|c|}{$\mathrm{xxxx}$} \\
\hline \multicolumn{4}{|c|}{ C.AITPOS OBRIGATORIO PAR.A PREENCHIMENTO } \\
\hline COMPONENTE & $\operatorname{sxxx}$ & CACSA & $\mathrm{xxxx}$ \\
\hline SLINTOMA: & XXXX & SERIIÇO & $\mathrm{XXXX}$ \\
\hline \multicolumn{4}{|c|}{ HORARIO DE MLIQUINA } \\
\hline PARADA & $\mathrm{XX}: \mathrm{XX}$ & LIBER+ÇÃO & $\mathrm{xX}: \mathrm{xx}$ \\
\hline INCIO D.AMALVTENÇ. IO & $\mathrm{XX}: \mathrm{XX}$ & FMDDAMANUTENC.io & $\mathrm{xx} \cdot \mathrm{xx}$ \\
\hline \multicolumn{4}{|c|}{ COMENT.ARIOS } \\
\hline PEÇA. & CODICO ALAOOXARIFADO & PECC.A & CODICO ALMIOX.ARIFADO \\
\hline $\operatorname{xxxx}$ & $\mathrm{xxxx}$ & & \\
\hline & & & \\
\hline
\end{tabular}

Fonte: Autores.

\section{Manutenção}

De acordo com Silva (2016) “objetivo da manutenção é prevenir todas as perdas causadas por problemas nos equipamentos." e complementa dizendo que "A equipe de manutenção devem assegurar que todos os equipamentos estejam em boas condições de operação.”. A parada para uma manutenção de qualidade deve proceder no menor tempo possível por técnicos qualificados que disponham das ferramentas adequadas para a execução 
do serviço.

O item 2.8 da NBR 5462/1994 define os termos relacionados com a confiabilidade e mantenabilidade, classificando os tipos de manutenção empregado. O item 2.8.7 define Manutenção Preventiva como: "Manutenção efetuada em intervalos predeterminados, ou de acordo com critérios prescritos, destinada a reduzir a probabilidade de falha ou a degradação do funcionamento de um item." O item 2.8.8 define Manutenção Corretiva como: "Manutenção efetuada após a ocorrência de uma pane destinada a recolocar um item em condições de executar uma função requerida". O item 2.8.9 define Manutenção Preditiva como: "Manutenção que permite garantir uma qualidade de serviço desejada, com base na aplicação sistemática de técnicas de análise, utilizando-se de meios de supervisão centralizados ou de amostragem, para reduzir ao mínimo a manutenção preventiva e diminuir a manutenção corretiva."

\section{PCM na indústria 4.0}

De acordo com Dias (2017) O termo "Indústria 4.0" começou a ser difundido em Hanover na Alemanha em 2011, entre investidores, representantes políticos e universitários, com o objetivo de alinhar e aperfeiçoar as tecnologias em desenvolvimento com o setor secundário alemão. Dias (2017) afirma que a fusão do mundo virtual com o real através de processos ciber-físicos serão extremamente necessários no desenvolvimento da indústria 4.0, e no surgimento de fábricas inteligentes capazes de monitorar e decidir virtualmente as variáveis da gestão. Lima (2019) define que o trabalho no setor de PCM ocorria inicialmente de forma manual com caneta e papel, se modernizando parcialmente ao longo dos anos com a inserção de computadores para auxiliar na organização e disposição de dados coletados.

Silva (2018) afirma que "a manutenção industrial tem avançado na aplicação de ferramentas de gestão de suas operações.”. Os gestores e demais envolvidos com o PCM da empresa coletam, armazenam e trabalham em cima de dados levantados nas Ordens de Serviço e desenvolvem eventuais diagnósticos que visam melhorar a produtividade. Silva (2018) salienta que "A indústria 4.0 projeta a manutenção preditiva como elemento de fundamental importância nesta mudança de paradigma.", em que o maquinário e os demais sistemas fabris terão inteligência capaz de expor em tempo real sua eficiência e necessidades de manutenção para seus gestores. A tabela 1 contém um comparativo entre a indústria 
vigente e a projeção da 4.0 em resumo (SILVA,2018).

Tabela 1: Vantagens da indústria 4.0 em relação ao modelo atual.

\begin{tabular}{|c|c|c|}
\hline INDÚSTRIA 3.0 & INDÚSTRIA 4.0 & VANTAGENS \\
\hline ESTOQUES CHEIOS & ESTOQUES DINÂMICOS & REDUÇÃO DE CUSTOS, \\
\hline $\begin{array}{c}\text { MANUTENÇÕES } \\
\text { PREVENTIVAS E CORRETIVAS }\end{array}$ & MANUTENÇÕES PREDITIVAS & $\begin{array}{l}\text { REDUÇÃO DE CUSTOS, } \\
\text { REDUÇÃO DE ERROS }\end{array}$ \\
\hline OPERÁRIO E MÁQUINA & MÁQUINA INTELIGENTE & $\begin{array}{c}\text { AUMENTO DE } \\
\text { SEGURANÇA, REDUÇÃO } \\
\text { DE ERROS. }\end{array}$ \\
\hline USINAGEM & IMPRESSÃO 3D & REDUÇÃO DE CUSTOS \\
\hline $\begin{array}{l}\text { COMUNICAÇÃO DE DADOS } \\
\text { VIA REDE /CABOS }\end{array}$ & BIG DATA & $\begin{array}{c}\text { SEGURANÇA, } \\
\text { TRANSPARENCIA NOS } \\
\text { NEGOCIOS }\end{array}$ \\
\hline MÃO DE OBRA & INTELIGÊNCIA & QUALIFICAÇÃO \\
\hline
\end{tabular}

Fonte: Adaptado pelos autores.

Borlido (2017) define fábrica inteligente como aquela que "trabalha na máxima eficiência enquanto as máquinas inteligentes interligadas entre si, colaboram entre elas, com os trabalhadores, com os fornecedores e clientes e com a cadeia analítica e dinâmica criada para se autocontrolar.". Neste contexto, muitas tecnologias emergentes vão ter aplicabilidade neste cenário em nova revolução industrial para com a gestão da manutenção, como a Realidade aumentada (RA), Nuvem de dados (Cloud), Internet das coisas (IoT - Internet of things) e Big Data. A figura 3 apresenta as eventuais mudanças ocasionadas pelas tecnologias emergentes.

Figura 3: Tecnologias na indústria 4.0 .

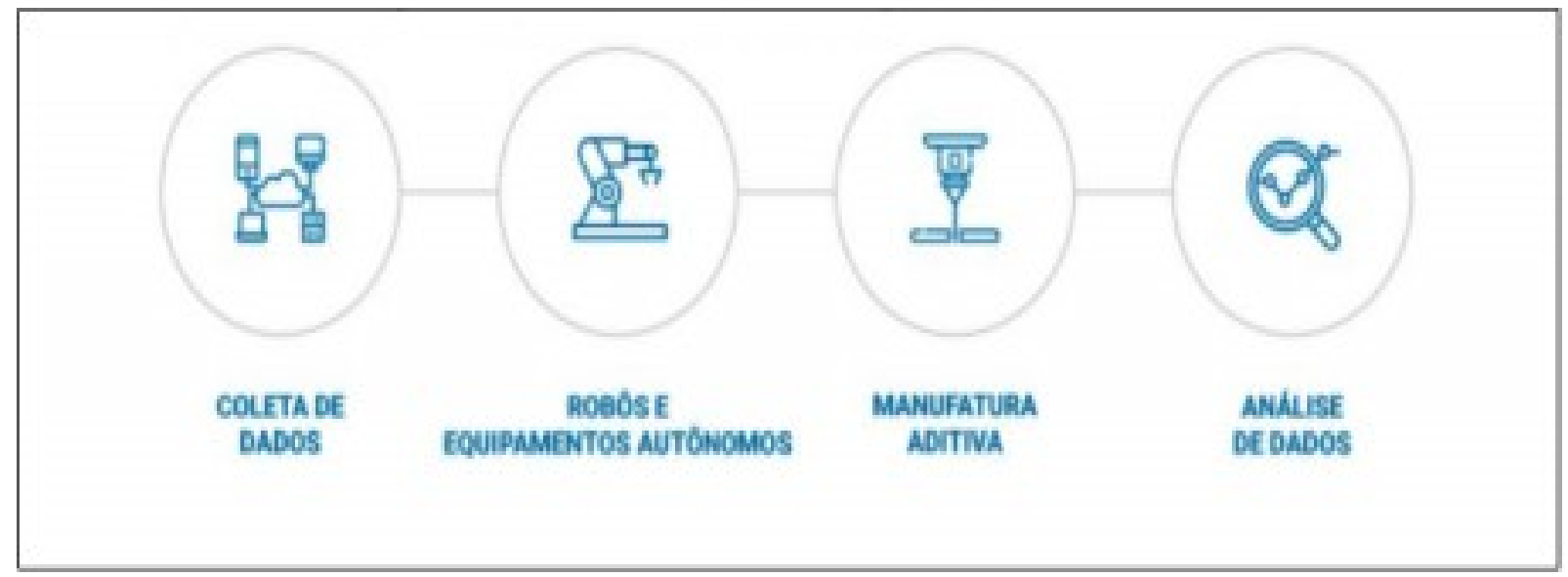

Fonte: A evolução da industria, Site da ROMI (2020). 
Realidade aumentada

Os sistemas com base em realidade aumentada são capazes de mensurar uma variedade de tarefas, desde a seleção de uma peça em um almoxarifado ou o envio de procedimentos de manutenção por meio de dispositivos móveis para um técnico em campo, tal ferramenta se torna indispensável (ALBERTO, 2018). Assim, com um mapeamento preciso em determinado maquinário ou processo, fica possível a realização de simulações que consigam precisar o local avariado, os suprimentos necessários para manutenção e eventuais procedimentos, facilitando o serviço do técnico na atuação do problema.

Com a realidade aumentada, a implementação de interfaces homem-máquina (IHMs) capazes de relacionar ativos de TI (Tecnologia da Informação) ao operador com feedbacks instantâneos acerca do estado do equipamento, geram confiabilidade para com os procedimentos a serem desenvolvidos na máquina. (ALBERTO, 2018). Essa comunicação física-digital entre operador e maquinário é indispensável na ambientação de indústria 4.0. Tal tecnologia encontra-se em etapa de desenvolvimento inicial, entretanto, a mesma será de muita utilidade no novo cenário industrial, visto que a confiabilidade e exatidão das informações vinculadas com a experiência do operador ou técnico responsável ocasionará em eficiência no exercício do trabalho. Na figura 4 tem-se demonstrado a aplicação de realidade aumentada no processo de manutenção de um veículo.

Figura 4: Realidade aumentada.

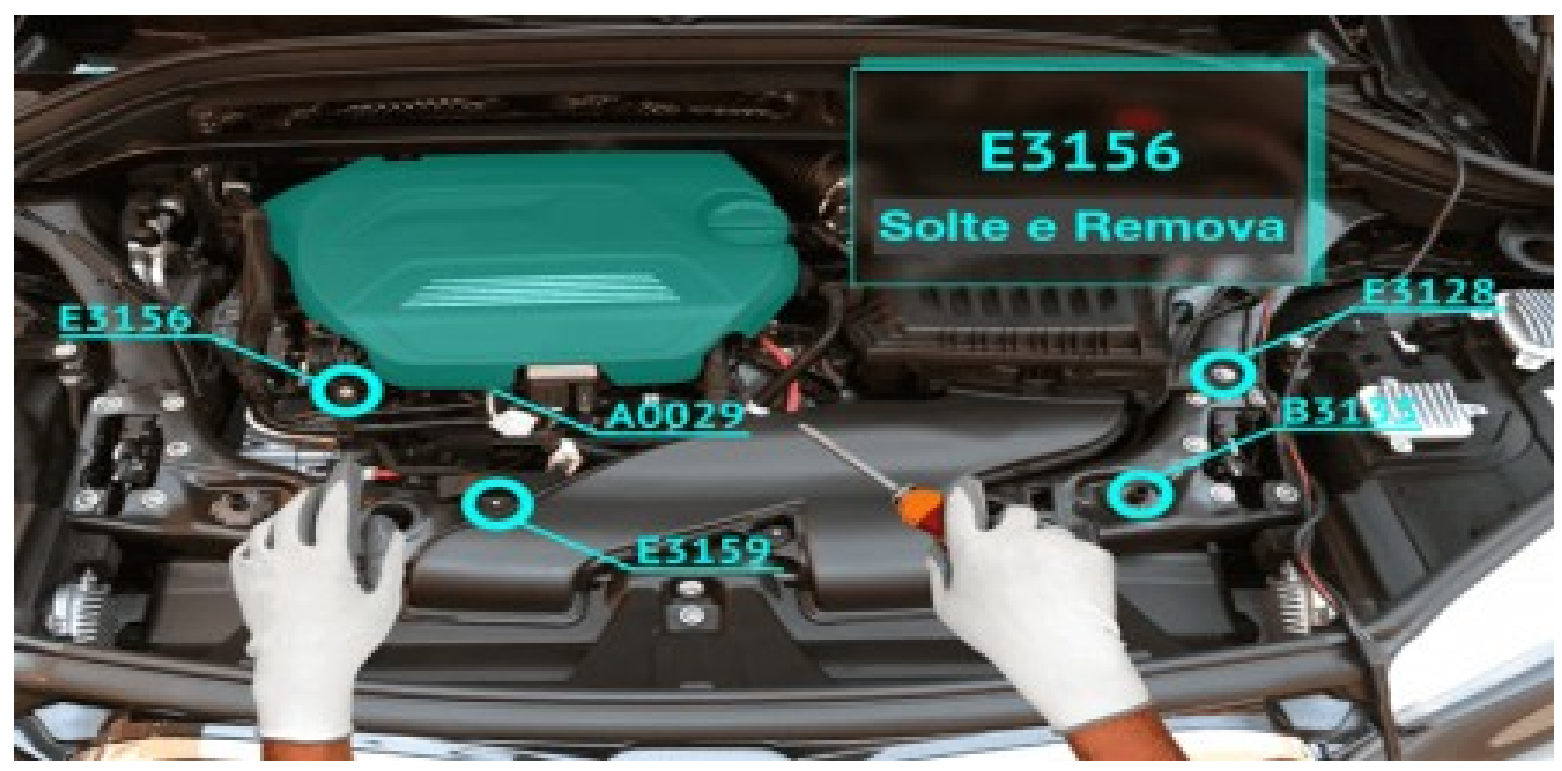

Fonte: Retirada do site VRMAT (2017). 
$\mathrm{Na}$ figura 4, o técnico que possui uma ferramenta com tecnologia de realidade aumentada realiza seu serviço de manutenção seguindo em tempo real todas as informações preexistentes e procedimentos necessários para o desenvolvimento de seu serviço.

Cloud (Nuvem de dados)

De acordo com Borlindo (2017), Cloud consiste no aproveitamento de memória, de armazenamento e cálculo em computadores e servidores interligados por meio de rede sem fio (wireless). A informação fica disponibilizada em uma "nuvem-compartilhada" (Cloud) com capacidade ilimitada de interconexão entre empresas. Dentre suas funções tem-se as opções de armazenar todos os dados empresariais com segurança, versatilidade e sincronismo automático, com acesso remoto por qualquer dispositivo vinculado a empresa, sendo também economicamente vantajoso para as organizações, que deixaram de investir em redes locais para interligação dos dados da empresa, como Switches, cabos de rede e computadores. Assim, todo o gerenciamento relacionado ao histórico de manutenções e organização de dados ficará arquivado virtualmente. A figura 5 apresenta a representação esquemática de interligação da nuvem.

Figura 5: Cloud.

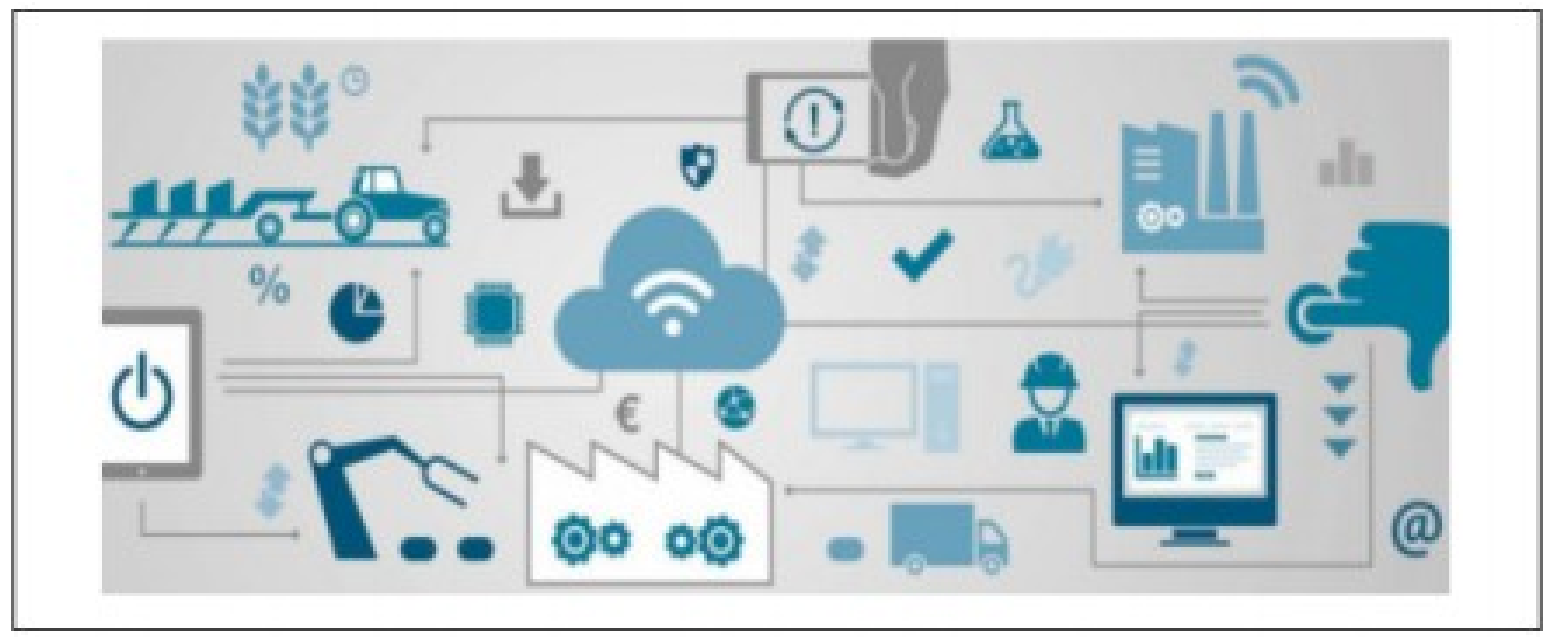

Fonte: Site Vende-se Máquinas (2017).

$\mathrm{Na}$ figura 5, tem-se representado a interligação de dispositivos inteligentes em uma nuvem de dados que relaciona todas as informações simultaneamente. 
Internet das coisas (IoT)

Galegale (2016) define Internet das Coisas (internet of things) como a rede mundial de objetos conectados, em que ocorre a troca de informação a nível global de maneira instantânea. Essa comunicação acontece por meio da interação cotidiana de equipamentos e dispositivos como sensores, transmissores e receptores existentes nas "coisas" que usamos que possibilitam a interação homem-máquina ou máquina-máquina por meio de rede internet.

De acordo com Borlindo (2017) no âmbito da indústria, tem como aplicabilidade primordial a informação e comunicação entre os dispositivos de detecção de dados (sensores e transdutores em geral) com atuadores (motores elétricos, válvulas etc) entre si ou entre as máquinas, proporcionando relatórios em tempo real de cada dispositivo isolado que fazem com que o próprio sistema se monitore e se controle. A figura 6 ilustra um comparativo com relação a Internet das coisas.

Figura 6: Indústria 3.0 e o cenário na 4.0.

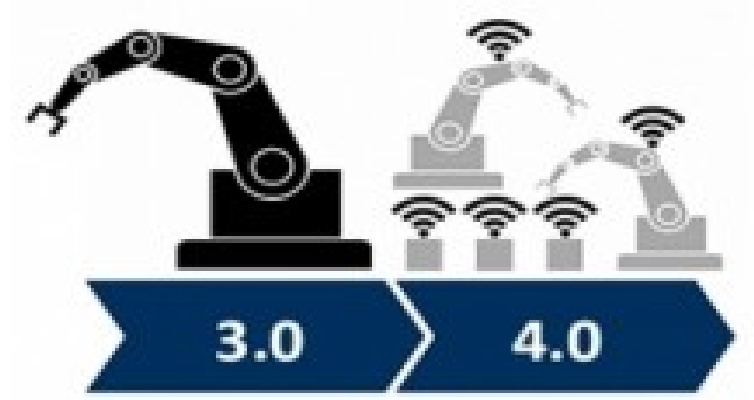

Fonte imagem: Site da Protec (2018).

A figura 6 apresenta um breve paralelo ilustrativo entre uma máquina na indústria 3.0 ( em comunicação de rede) com uma máquina na indústria 4.0 (Com tecnologia de IoT).

Big data

Conforme Mello (2015) retrata em seu artigo, o Big data é uma tecnologia utilizada por gestores que almejam versatilidade na tomada de decisões, capaz de processar uma infinidade de dados simultaneamente, com confiabilidade entre suas informações, sendo também conhecido como $3 \mathrm{Vs}$, por relacionar o volume de grande quantidade de dados, com uma velocidade em tempo real, independente da variabilidade do dado em relação a origem e estrutura. A figura 7 demonstra a correlação entre os 3Vs. 
Figura 6: Big Data.

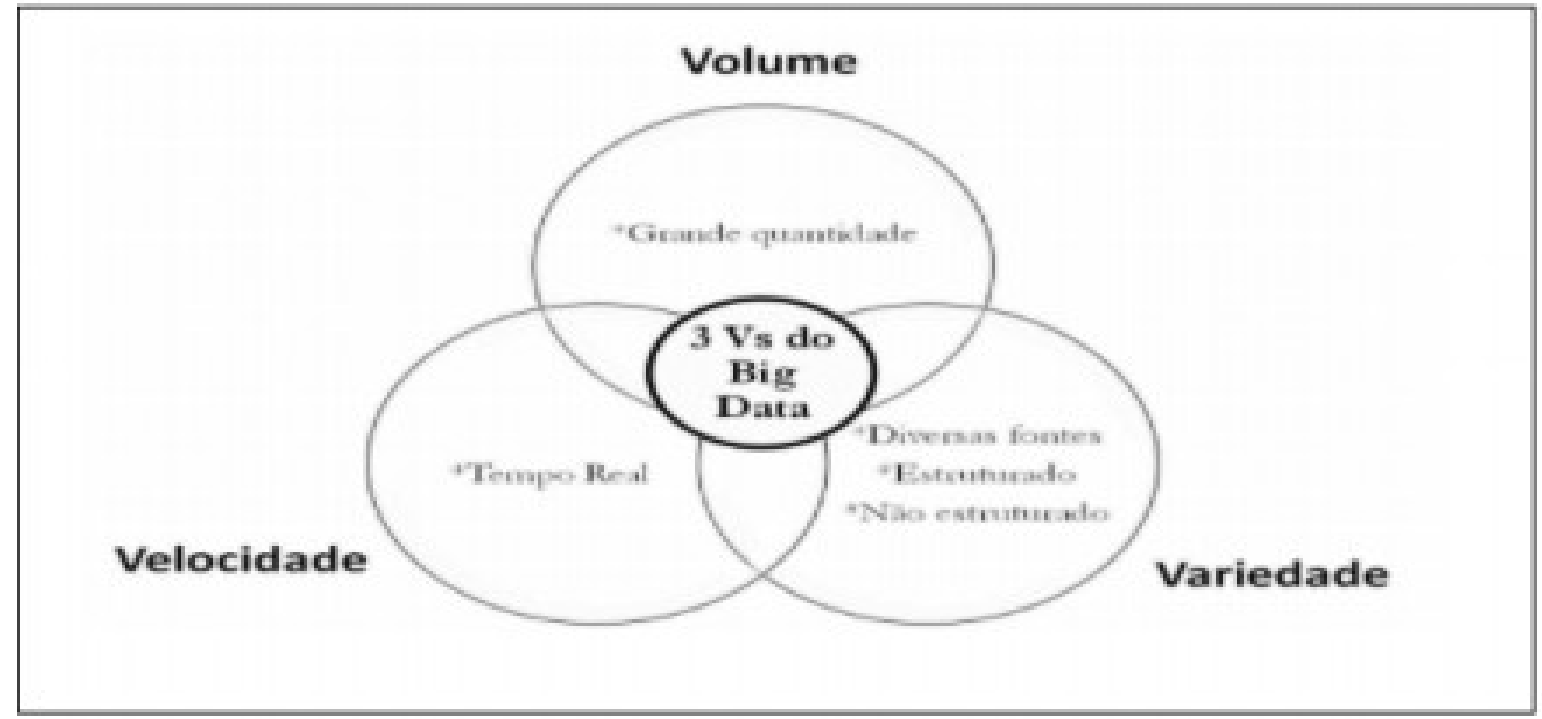

Fonte: Mello (2015)

A figura 7 apresenta os pilares da tecnologia Big Data, com relação ao dinamismo empregado.

\section{CONCLUSÃO}

Com o estudo comparativo, apresentado no item 3, conclui-se portanto que a gestão manutenção na indústria 4.0 vem para auxiliar de maneira dinâmica o funcionamento contínuo do setor secundário, para com a necessidade de atender a demanda produtiva da sociedade por produtos de alta qualidade, baixo custo e menor tempo para fabricação, sendo que para tal feito, é indispensável a utilização das tecnologias emergentes.

Buscando atender esta clientela diversificada na atualidade e no futuro, as empresas devem se reinventar, melhorando continuamente seus sistemas organizacionais e de gestão, acompanhando as tecnologias existentes e em desenvolvimento no mercado, e estudando maneiras de implementar as mesmas em seu cotidiano empresarial, para assim alcançarem seus objetivos.

A indústria 4.0 já começou, e as empresas não devem decidir se vão ou não entrar nesta realidade, mas sim como vão fazer para entrar e operar aos novos moldes, visto que as novas tecnologias estão se desenvolvendo em ritmo acelerado e difícil de se acompanhar, a ponto de uma quinta revolução venha a ocorrer antes que a quarta seja implementada em todo o mundo. 


\section{REFERÊNCIAS}

ALBERTO, A; Lima, T.D.F.M.; Charrua-Santos, F.M.B. ; Santos, B.P. .INDÚSTRIA 4.0: DESAFIOS E OPORTUNIDADES. Huambo, Angola, 2018 (Artigo, Universidade José Eduardo dos Santos).

BORLINDO, David José Araújo, Indústria 4.0 - Aplicação a Sistemas de Manutenção. Porto, Portugal, 2017 (Dissertação de mestrado, Faculdade de engenharia ,Universidade do Porto).

CAMPOS, Ronaldo Ribeiro de; SANITÁ, William Matheus, PCM, planejamento e controle da manutenção. Disponivel em https://revista.fate ctq.edu.br/index.p hp/interfacetecnol ogica/article/view/791/505 acesso em 25 de Outubro de 2020.

DIAS, Ana Valéria Carneiro; SILVA, Pedro Paulo; TROPIA, Célio Eduardo Zacharias, INDÚSTRIA 4.0: UMA CARACTERIZAÇÃO DO SISTEMA DE PRODUÇÃO. Disponível em https://www.researchgate.net/public ation/317475373_Indust_ria_40 _um a_caracterizacao_do_sistema_de_producao acesso em 25 de outubro de 2020.

FILHO, G. B. A organização, o planejamento e o controle da manutenção. Rio de Janeiro,Editora Ciência Moderna Ltda.; 2008.

GALEGALE, Gustavo Perri; SILVA, Carolina Bertolucci Hilário e; SIQUEIRA, Érica; SOUZA, Cesar Alexandre de. INTERNET DAS COISAS APLICADA A NEGÓCIOS - UM ESTUDO BIBLIOMÉTRICO. São Paulo, Brasil, 2016 (Artigo, Universidade de São Paulo-USP).

KON, Anita. NOVA ECONOMIA POLÍTICA DOS SERVIÇOS . São Paulo, Brasil, 2015 (Livro).

LIMA, Elon Vieira; SILVA, Daniela dos Santos da, O PLANEJAMENTO E CONTROLE DA MANUTENÇÃO NA INDÚSTRIA 4.0 . Ponta Grossa, 2019. Disponivel em http://aprepro.org .br/conbrepro/2019/anais/a rquivos/09302019_100936_5d920250791a4.pdf acesso em 25 de Outubro de 2020.

MELLO, Raquel Gama Soares de. UTILIZAÇÃO DE BIG DATA ANALYTICS NOS SISTEMAS DE MEDIÇÃO DE DESEMPENHO: ESTUDO DE CASO. São carlos, Brasil, 2015. ( Dissertação de Mestrado, Universidade Federal de São Carlos).

MENELAU, Sueli; MACEDO, Francisco Guilherme Lima; CARVALHO, Patrícia Lacerda de; NASCIMENTO, Thiago Gomes; JÚNIOR, Antônio Deusany de Carvalho. Mapeamento da produção científica da Indústria 4.0 no contexto dos BRICS: reflexões e interfaces. Cadernos EBAPE.BR. 2019, (FGV, Fundação Getulio Vargas).

Norma NBR 5462/2014 disponível em https://pt.slideshare.net/eavargas2512/nbr-5462-2 acesso em 26 de outubro de 2020.

PROTEC, Pró Inovação na Industria Brasileira,disponivel em http://www.protec.org.br/politicas-publicas-e economia/36503/fomento-a-industria-4.0-e-internet-das-coisas-precisa-avancar, acesso 01 de novembro de 2020. Figura 6

ROMI, Industrias ROMI S.A. Disponivel em https://www.romi.com/a-evolucao-da-industria-entenda-o-im pacto-da-industria-4-0/ acessado 01 de 
Novembro de 2020 . Figura 3

SILVA, Maria Inês Tavares, Melhoria de processos e manutenção inteligente de ferramentas no âmbito do industria 4.0 . Porto, Portugal, 2016 (Dissertação de mestrado, Faculdade de engenharia ,Universidade do Porto).

SILVA, Edson Pereira da, A TRANSIÇÃO DA MANUTENÇÃO INDUSTRIAL PARA O MODELO DO NOVO PARADIGMA DA INDÚSTRIA 4.0 . São Paulo, 2018 (Dissertação de Mestrado, Programa de pós-graduação em engenharia de produção, Universidade Paulista-UNIP).

VENDE-SE MAQUINAS, disponível em

http://www.web.vendesemaquinas.com.br/noticias/industria-4

-0-jornada-para-cloud-com-transformacao-digital-8e3e acesso 01 de novembro de 2020. Figura 5

VIANA, H. R. G. PCM: planejamento e controle da manutenção. Rio de Janeiro:Qualitymark, 2002. Figura 1

VRMAT, VRMAT building Virtual knowledge,disponivel em http://www.vrmat.com.br/pt /realidade -aume $\mathrm{n}$ t ada-manutencao-industria/ acesso 01 de novembro de 2020. Figura 4. 